\title{
Development of a methodology for data validation in power systems using different types of measurements
}

\author{
Pavel Y. Kovalenko ${ }^{1}$, Valentin I. Mukhin ${ }^{1, *}$, and Mihail D. Senyuk ${ }^{1}$ \\ ${ }^{1}$ Ural Federal University, Automated electrical systems department, 620002, Yekaterinburg, Russia
}

\begin{abstract}
In recent years, the technology of synchrophasor measurements has been introduced in power systems around the world. When managing a power system and predicting its operation conditions, the important task is to check the validity of power system data. At the same time, the traditional types of measurements, such as digital fault recorders and telemetry devices are still widely used. It is known that redundancy of measurements contributes to a more accurate solution of the data validation problem. It is useful to create a method for data validation in power systems, which could involve various types of measurements in order to increase the redundancy and, hence, the overall accuracy of measurements. This study presents some validity criteria that use the idea described above. The results of testing the proposed methodology on the substation model in Matlab software package are presented and discussed.
\end{abstract}

\section{Introduction}

A generally recognized effective way to ensure the reliability of information at power facilities is to increase the redundancy of measurements, because with an increase in the number of measurements, the probability of the presence of unreliable measurements decreases. The study proposes a method for verifying data inside a power facility using different types of measuring instruments. This is a good way to achieve redundancy in measurements, which is not always possible with techniques based on the use of any one type of measurement.

To work with different types of measuring instruments, an appropriate information platform will be required, the creation of which is quite realistic at the modern level of technology development [1]. According to the NASPI report [2], it becomes feasible to collect and process point-on-wave measurements of the parameters of the power system operation conditions. Given the large volume of such information, its constant transfer to a higher level of management system is not expected. Therefore, in this case it would be optimal to solve the problem of data validation within a power facility.

This article begins a series of studies devoted to investigating the possibility and feasibility of using point-on-wave measurements of electrical parameters to solve the problem of data validation in electrical power systems. The study [3] provides an overview of methods for data validation [4-9] obtained from devices for synchronized phasor measurements. In addition to those, other research works on similar topics were studied [1017], but the idea of using different types of measurements in order to increase data redundancy to solve the problem of validation was not described.

\section{Proposed methodology}

\subsection{Definition of a set of measurement}

This study assumes the following set of measurements is involved:

- measurements of phasors and point-on-wave values of currents in phases are on the overhead line;

- measuremens of phasors and point-on-wave values of currents in phases on high and medium voltage windings and the magnitudes of the current in the common neutral wire of the high and medium voltage windings, magnitudes and point-on-wave values of the low voltage winding currents are on the autotransformer;

- measurements of phasors and point-on-wave values of phase-to-phase voltages are on the busbar system.

\subsection{Formulation of validity conditions}

The use of this set of measurements makes it possible to formulate the following conditions for the absence of unreliable measurements.

- The first Kirchhoff rule on the bus system is satisfied simultaneously by point-on-wave values of currents and by phasors of currents in each phase

$$
\sum_{i} I_{i}^{\text {Point-on-wave }}=0 \bigcap \sum_{i} I_{i}^{\text {Phasor }}=0
$$

\footnotetext{
*Corresponding author: valentinmukhin@yandex.ru
} 
- The first Kirchhoff rule in each phase is satisfied for point-on-wave values of currents and balance of phase-to-phase voltages on the busbar system

$$
\sum_{i} I_{i}^{\text {Point-on-wave }}=0 \cap U_{A B}=U_{B C}=U_{C A}
$$

- The first Kirchhoff rule in each phase is satisfied for phasors of currents and balance of phase-to-phase voltages on the busbar system

$$
\sum_{i} I_{i}^{\text {Phasor }}=0 \cap U_{A B}=U_{B C}=U_{C A}
$$

- On transformers - the equality of the magnitudes of the reduced currents phasors on the high-voltage side and currents phasors on the low-voltage side in phases

$$
\begin{aligned}
& \dot{I}_{1 A}^{\prime}=\dot{I}_{2 A} \\
& \dot{I}_{1 B}^{\prime}=\dot{I}_{2 B} \\
& \dot{I}_{1 C}^{\prime}=\dot{I}_{2 C}
\end{aligned}
$$

- Equality of the magnitudes of the phasor sum of the phase currents and the magnitudes of the current in the neutral wire in the star of the transformer

$$
\operatorname{abs}\left(\dot{I}_{A}+\dot{I}_{B}+\dot{I}_{C}\right)=I_{N}
$$

In case any of these conditions ceases to be true, it drives a conclusion that there is at least one unreliable measurement in the set.

\section{Testing}

\subsection{Test scheme description}

A 330/220/110/10 kV substation was used as a test scheme. The schematic diagram of the substation under consideration is shown in Fig. 1.

Twelve overhead lines (L1-L12) are connected to the substation. Autotransformers (AT1-AT4) are used to connect switchgears of various voltage classes. The substation has reactive power compensation devices: two synchronous compensators ( $\mathrm{SC} 1, \mathrm{SC} 2)$ and two static capacitor banks (SCB1, SCB2). In addition, a gas turbine power plant (GTPP) with a total installed capacity of 135 $\mathrm{MW}$ is connected to the $110 \mathrm{kV}$ switchgear.

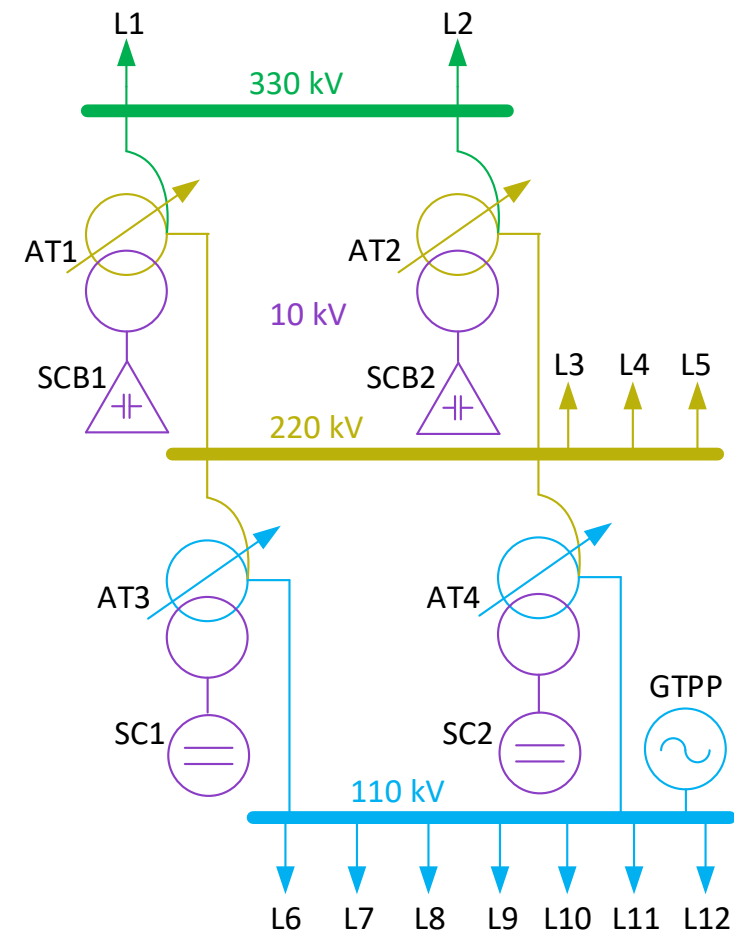

Fig. 1. Scheme of the substation under consideration.

\subsection{Simulation aspects}

To carry out computational experiments, a model of the considered substation was created in Matlab Simulink software. Loads and static capacitor banks are modelled using "Three-Phase Series RLC Load" blocks, synchronous generators, synchronous compensators, as well as overhead lines that supply the substation are modelled using "Three-Phase Source" blocks, autotransformers are modelled using "Three-Phase Transformer Inductance Matrix Type (Three Windings)".

Three types of measurements are also implemented in the substation model.

- Digital fault recorder (DFR) which provides instantaneous values of measured values with a predefined sampling frequency. Simulation of this type of measurements does not require the use of additional blocks, because the "Continuous" simulation type is selected in the "PowerGUI" block. "To Workspace" blocks are used for data transfer to Workspace. In the "Sample time" field of these blocks, one should input a value that is the reciprocal of the required sample rate.

- Phasor measurement unit (PMU) that provides the amplitude and phase of the measured sinusoidal signal. To simulate this type of measurement, "Fourier Analysis" blocks are used, which require a fixed frequency of $50 \mathrm{~Hz}$. Also, "Magnitude-Angle to Complex" blocks were used to transfer complex values to Workspace. The magnitude was divided by the square root of 2 according to the standard [18]. The implementation of this measuring device in the Matlab Simulink software is shown in Fig. 2. 


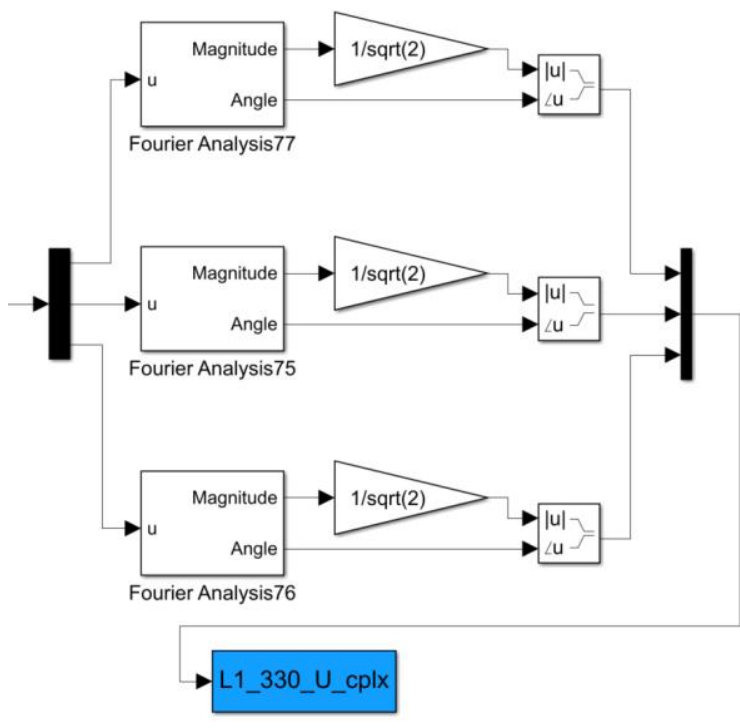

Fig. 2. The implementation of this measuring device in the Matlab Simulink software.

- The device of traditional SCADA-measurement, which provides the magnitude (effective value) of the measured value. To simulate this type of measurement, the signal amplitude values from the "Fourier Analysis" blocks are used, divided by the square root of 2 .

\subsection{Test results}

The testing was carried out as follows. The substation was modelled, after which it was equipped with measurements, as well as blocks for outputting data to Workspace. After that, a simulation was run with a duration of 0.5 seconds. Since the sampling frequency was set equal to $10 \mathrm{kHz}$, as a result of simulation, 5000 samples of parameter values were obtained from each measuring device.

In practice, PMUs calculate phasor parameters based on point-on-wave values. Point-on-wave values are not transmitted outside the PMU. In addition, the DFR devices, which allow obtaining point-on-wave values of the electrical parameters, in most cases are not synchronized with each other. For research purposes, the assumption was adopted that DFR devices are synchronized and independent of PMU measurements. It should be noted that the implementation of such an approach at the present stage of technology development seems feasible.

Fig. 3 shows a graph of the value of the sum of pointon-wave values of currents in the $330 \mathrm{kV}$ busbar system. As one can see from the figure, the sum of the currents throughout the simulation is close to zero. Hence, one can conclude that the first Kirchhoff rule is fulfilled with some permissible error.

The case where the measuring device starts sending invalid data is illustrated in Fig. 4. As we can see from this figure, the first Kirchhoff rule is satisfied for the first half of the simulation time. Starting from $0.25 \mathrm{~s}$, a data error is simulated by increasing the measured current value in L1 by $10 \%$. Failure to comply with the first
Kirchhoff rule in the second half of the simulation time indicates the presence of invalid data.

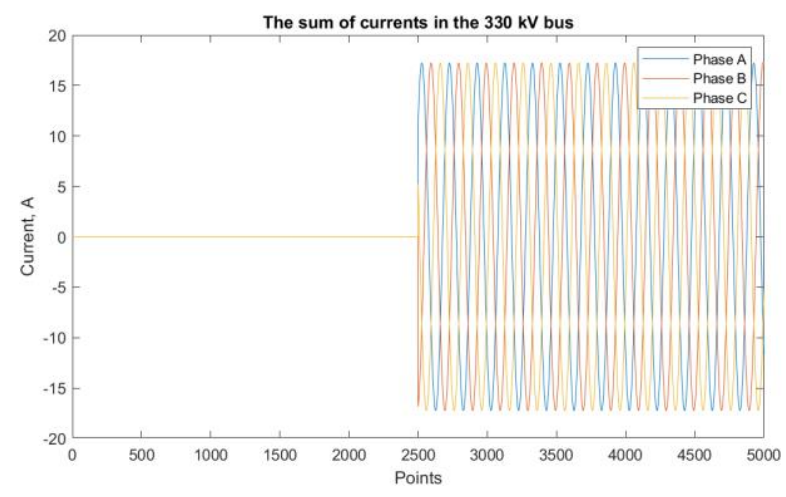

Fig. 3. The sum of currents in the $330 \mathrm{kV}$ node when the first Kirchhoff rule is satisfied.

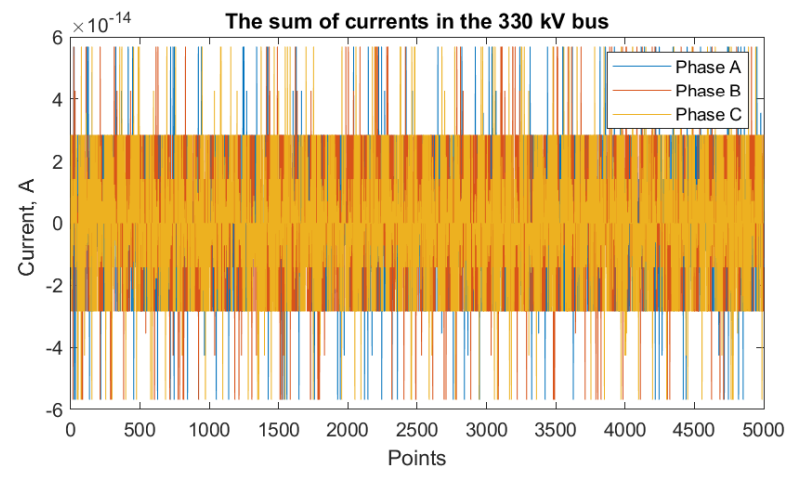

Fig. 4. The sum of currents in the $330 \mathrm{kV}$ node upon occurrence of invalid data at $\mathrm{t}=0.25 \mathrm{~s}$.

\section{Discussion}

In case of the proposed methodology implementation, it should be taken into account that it is impossible to achieve absolutely exact fulfillment of the validity conditions (1) - (5). This is due to the errors of the measuring equipment, as well as various accompanying phenomena, for example, the flow of the magnetizing current in the power transformer for the validity condition (4). In further research, it will be necessary to determine the permissible degrees of unvalidity and permissible errors, under which the validity conditions can be considered fulfilled. For this purpose, real data sets will be used.

It should be noted that the synchrophasor measurement data validation system must perform automatic periodic validation of the synchrophasor measurement data to signal the possibility of the following problems:

- failure of the measuring transducer. The measuring transducer is assumed to have failed if it cannot be connected to;

- measurement out of the accuracy class of the measuring transducers. It is assumed that there is a failure in the secondary circuits of measuring transformers, if the connection to the measuring transformer is established, it does not transmit error messages, but the measurement values are absent or 
close to 0 due to electromagnetic interference, etc., while it is reliably known that the connection is not disabled;

- software failure of the measuring transducers. Transmitter software is presumed to have failed if it is connected but is reporting an error;

- incorrect phase sequence when connecting voltage circuits. With correct phase rotation, the angle difference between the voltage vectors $\mathrm{A}-\mathrm{B}$ and $\mathrm{C}-\mathrm{A}$ should be equal to $120^{\circ}$. If the alternation is wrong, the same differences will be equal to $120^{\circ}$;

- wrong polarity of current circuits connection. The polarity of the current connections is easy to track for generator connections and power transmission lines leaving power plants. On the other hand, power transmission lines between two substations may have reversible power flows. In this case (under the assumption of a possible incorrect polarity of the connection of the current circuits of only one connection), it can be detected by calculating the power balance over the buses.

It is worth noting that it is very helpful to receive discrete signals, telemetry data, and telesignalling detect inaccuracy. This will contribute, in particular, to an understanding of the state of the switching devices at a given time.

\section{Conclusion and future work}

This study shows the idea of a method for verifying data within a power facility by increasing the redundancy of measurement information. Increased redundancy can be achieved by using data obtained simultaneously from different sources. The article proposes conditions for reliability. Their testing was carried out on a substation model in Matlab Simulink software. As for further work, it is planned to develop new validity conditions in addition to those already existing, and to check the operation of these conditions on real data. It is also necessary to develop the functionality of the validation system, which would make it possible to identify not only the fact of occurrence of an inaccurate measurement, but also the inaccurate measurement itself. For this purpose, it is proposed to use any of the existing methods of data validation. At the same time, it will need to be modified to be able to work with different types of measured data. In addition, a detailed study of the technical side of the synchronization of time data obtained from various sources is required. This will also be the subject of further research.

\section{References}

1. M. Kezunovic, C. Zheng, C. Pang, Operational, and Non-Operational Data for Interpreting Alarms, Locating Faults and Preventing Cascades, 2010 43rd Hawaii International Conference on System Sciences (2010)

2. A. Silverstein, Dr.J. Follum, High-Resolution, Time-Synchronized Grid Monitoring Devices, North American Synchrophasor Initiative (NASPI) Technical Report (2020)
3. P. Kovalenko, V. Mukhin, M. Senyuk, D. Kornilova, Review of Methods for Power Systems State Estimation and Data Validation Based on Synchrophasor Measurements, 2020 IEEE 61th International Scientific Conference on Power and Electrical Engineering of Riga Technical University (RTUCON) (2020)

4. I. Idehen, T. Overbye, A similarity-based PMU error detection technique, 2017 19th International Conference on Intelligent System Application to Power Systems (ISAP) (2017)

5. Z. Mao, T. Xu, T. Overbye, Real-time detection of malicious PMU data, 2017 19th International Conference on Intelligent System Application to Power Systems (ISAP) (2017)

6. I. Idehen, Z. Mao, T. Overbye, An emulation environment for prototyping PMU data errors, 2016 North American Power Symposium (NAPS) (2016)

7. I. Idehen, T. Overbye, PMU time error detection using second-order phase angle derivative measurements, 2019 IEEE Texas Power and Energy Conference (TPEC) USA (2019)

8. Q. Zhang, X. Luo, D. Bertagnolli, S. Maslennikov, B. Nubile, PMU data validation at ISO New England, 2013 IEEE Power \& Energy Society General Meeting (2013)

9. F. Wang, Q. Liu, F. Xiong, L. Guo, J. Feng, Q. Wang, Data validation and anomaly detection techniques for smart substations, 2017 IEEE Conference on Energy Internet and Energy System Integration (EI2) (2017)

10. I.N. Kolosok, E.S. Korkina, A.E. Mahnitko, Detection of systematic errors in PMU measurements by the power system state estimation methods, 2015 56th International Scientific Conference on Power and Electrical Engineering of Riga Technical University (RTUCON) (2015)

11. A. Rouhani, A. Abur, Local detection of PMU measurement errors using dynamic state estimators, 2017 IEEE Power \& Energy Society Innovative Smart Grid Technologies Conference (ISGT) (2017)

12. R. Nair, P. Babu, A method for error detection and correction of the PMU measurements, 2014 International Conference on Computation of Power, Energy, Information and Communication (ICCPEIC) (2014)

13. D. Shi, D.J. Tylavsky, N. Logic, An adaptive method for detection and correction of errors in PMU measurements, IEEE TSG, 3, 1575-1583 (2012)

14. L. Zhang, A. Abur, Impact of tuning on bad data detection of PMU measurements, IEEE PES Innovative Smart Grid Technologies (2012)

15. A. Karpilow, R. Cherkaoui, S. D'Arco, T.D. Duong, Detection of bad PMU data using machine learning techniques, 2020 IEEE Power \& Energy Society Innovative Smart Grid Technologies Conference (ISGT) (2020) 
16. K. Mahapatra, N.R. Chaudhuri, R. Kavasseri, Online bad data outlier detection in PMU measurements using PCA feature-driven ANN classifier, 2017 IEEE Power \& Energy Society General Meeting (2018)

17. J.M. Lim, C.L. DeMarco, Bad data detection and estimation in high dimensional measurement data, 2017 IEEE Power \& Energy Society General Meeting (2018)

18. IEEE Standard for Synchrophasor Measurements for Power Systems, IEEE Std C37.118.1 ${ }^{\mathrm{TM}}-2011$ (2011). 